\title{
The Change in Pulse Rate and Behavioral Score by Using Video Assisted Induction of Pediatric Anesthesia
}

\author{
Yukako Abukawa ${ }^{1 *}$, Koichi Hiroki',2, Makoto Ozaki ${ }^{1}$ \\ ${ }^{1}$ Department of Anesthesia and Critical Care, Tokyo Women's Medical University, Tokyo, Japan \\ ${ }^{2}$ Kanagawa Children's Medical Center, Yokohama, Japan \\ Email: *yukakoabukawasandy@yahoo.co.jp
}

Received 22 January 2016; accepted 28 March 2016; published 31 March 2016

Copyright (C) 2016 by authors and Scientific Research Publishing Inc.

This work is licensed under the Creative Commons Attribution International License (CC BY). http://creativecommons.org/licenses/by/4.0/

c) (i) Open Access

\begin{abstract}
Purpose: Objective of this study was to determine whether video assisted anesthesia induction reduced pediatric patients' stress. Methods: With approval from the local ethics committee and parental informed consent, 75 children undergoing minor surgery were investigated in this prospective observational study. Patients were divided into three groups: group 1 was aged two to three years old, group 2 was aged four to six years old and group 3 was aged from seven to ten years old. The following three characteristics were evaluated: 1) the pulse rate at four points (the ward, the entrance at the operating room, mask notification and the mask fit); 2) the behavioral score in the operating room; 3 ) the amount of pain killers after the operation. Results: In group 1 $(\mathrm{N}=20)$, there was a significant difference between the control group and the video assisted group regarding the percentage change in pulse rate based on the children's ward when the patients looked at the mask. In group $2(\mathrm{~N}=26)$, there was no significant difference regarding any points. In group $3(\mathrm{~N}=29)$, there was a significant difference between control and video assisted group regarding the percentage change in pulse rate based on the children's ward for all points. Also, regarding to the behavioral score, there was a significant difference between the control group and the video assisted group of all ages. However, there was no significant difference regarding the use of NSAIDs in the postoperative period between the control and the video assisted group. Conclusion: These results show that the video assisted anesthesia induction is effective for pediatric patients.
\end{abstract}

\section{Keywords}

Video Assisted Anesthesia Induction, Pulse Rate, Pediatric General Anesthesia

\footnotetext{
${ }^{*}$ Corresponding author.
}

How to cite this paper: Abukawa, Y., Hiroki, K. and Ozaki, M. (2016) The Change in Pulse Rate and Behavioral Score by Using Video Assisted Induction of Pediatric Anesthesia. Open Journal of Anesthesiology, 6, 45-50. 


\section{Introduction}

Zeev N. demonstrated that reducing the level of preoperative anxiety before surgery would lead to a decrease in preoperative anxiety and fewer postoperative analgesic requirements [1]. Advanced preoperative preparations program, which was the anxiety reduction, distraction, video modeling and education, adding parents, no excessive reassurance, coaching, and exposure/shaping, reduced children's stress rather than the children in the control group.

Children might vocalize their fears with crying and agitation. This was accompanied by significant physiological changes such as increase in heart rate and secretion of stress hormones. There were some risk factors. One was the children's factor and the other was perioperative environmental factors. The children's factors were age, temperament, previous medical encounters, attachment style and quality of parent-child relationship. From 9 months, there might present separation anxiety and peaked at 1 year old.

Other factors were perioperative environmental factors, as increased levels of anxiety in children were associated with the number of people in the room at induction of anesthesia. Anesthesia induction was known to be the most anxiety provoking part of the preoperative experience.

In pediatric patients' slow induction, the children were anesthetized through a mask. We thought that the mask would cause stress for the children. The objective of this study was to determine if video assisted anesthesia inductions reduced the children's stress regarding a decrease in pulse rate and the behavioral score in pediatric patients.

\section{Methods}

With approval from the local ethical committee and parental oral and written informed consent, ASA physical states score I-II, seventy five children from two to ten years old were included in this prospective observational study. Exclusion criteria included patients having mental retardation, a developmental delay, and having received several operations under general anesthesia. The patients were divided into three groups: group 1 was aged two to three years old, group 2 was aged four to six years old, and group 3 was aged seven to ten years old. All patients underwent preoperative fasting. The operation time was estimated to be less than 3 hours. The following three characteristics were evaluated: 1 ) the pulse rate at four points (the children's ward, the entrance at the operating room, at the mask notification, and the mask fit); 2) the behavioral score at four points; 3) the amount of painkillers on the postoperative day. We evaluated the percentage of pulse rate based on the children's word. The pediatric patients were divided into two groups: the control group and video assisted induction group by the envelop methods to select the randomization to removing selection bias.

No patients received premedication. In the children's ward, the nurse measured the pulse rate by the oximetry monitor. At the entrance to the operation room, children in the video assisted induction group chose a DVD by themselves from twelve different kinds of DVD. In group 1 (age two to three years old), it was difficult to choose the DVD by themselves so the parents helped them in choosing a DVD. Then the pulse oximetry monitor was worn on the patients' finger. They could watch the DVD from the entrance to the operating room. In the control group, the only children entered the operating room without a DVD player. The children were separated with their parents at the entrance of operating room. From the entrance of operating room, after the children were on the operation table, we measured the pulse rate again by pulse oximeter. However, we did not use the non-invasive blood pressure monitoring and electrocardiography at this point. Then we talked about the face mask, which was soft, and showed the mask to the children. At this point, we measured the pulse rate as they looked at the mask. After we discussed the mask, the children attached the mask to their face. At the same time, the pulse rate was measured as the mask was fit. At this point, patients in haled only the oxygen even if slow induction was chosen. From at the entrance of operating room, the supervisor anesthesiologist checked the behavioral score. The behavioral score was chosen the number of distress behaviors: crying, screaming, nonverbal resistance and verbal resistance as Chorney previously described [2]. Standard monitoring such as non-invasive blood pressure monitoring, electrocardiography, capnography (Compact airway modules E-CAiOV, GE Heathcare, NJ) and bispectral index was applied after they lost consciousness by general anesthesia. Anesthesia induction was standardized as above, but was left to the discretion of the experienced pediatric anesthesiologists using either propofol or sevoflurane induction. After the operation, the pediatric patients received painkillers NSAIDs prospectively. 


\section{Statistics}

Data were expressed as the mean \pm standard deviation (SD). P values $<0.05$ were considered statistically significant. Statistical analysis was performed using the Sigma plot 13 software package. For the comparison between the control group and the video assisted induction group in each age group, all pairwise multiple comparisons were analyzed with the Holm-Sidak method.

\section{Results}

We studied seventy five children. We excluded one child in group 1 since that child refused wearing the pulse oximetry monitor. The patients' characteristics were shown in Table 1 . Body weight's mean \pm SD in group 1 were $14 \pm 2$ and $13 \pm 2$ (control group and video assisted induction group respectively) Body weights in group 2 were $17 \pm 2$ and $17 \pm 3$. Body weights in group 3 were $25 \pm 6$ and $27 \pm 5$. Heart rates at the children's ward in group 1 were $95 \pm 15$ and $96 \pm 12$. Heart rates at the children's ward in group 2 were $89 \pm 9$ and $90 \pm 17$. Heart rates at the children's ward in group 3 were $83 \pm 12$ and $87 \pm 18$. There were no difference between control group and Video assisted induction group regarding to each characteristic parameters in every groups. Table 2 showed that the kinds of operations. The stresses of the operations were difference so that some of children were taken not only the general anesthesia but also the caudal epidural anesthesia. First, we described the change in the pulse rate (Figure 1). In group 1, there was a significant difference between the control group and the video assisted induction group regarding the change in the pulse rate upon seeing the mask (mean \pm SD were $37.5 \pm$ 30.0 vs. $14.0 \pm 12.7, \mathrm{p}=0.015$ ). In group 2 , there was no difference between groups at any point. In group 3 , there was a significant difference between the control group and the video assisted group regarding the change in the pulse rate at all points $(15.9 \pm 14.7$ vs. $1.9 \pm 17.8,23.4 \pm 19.4$ vs. $1.1 \pm 19.1$ and $16.2 \pm 14.5$ vs. $2.0 \pm 17.9$, $\mathrm{p}=0.008,<0.001,0.008$ respectively). Second, we described the behavioral score for every group (Figure 2 ). There was significant difference between the control group and the video assisted group for all groups. However, there was no significant difference regarding the use of NSAIDs in the postoperative period between the control and the video assisted group (Table 3 ). The percentages of NSAIDs usage were $13 \%$ in control group versus $17 \%$ in video assisted induction group.

Table 1. This table showed that the patients’ physical data which were age, body weight, height and pulse rate in each group. There was no significant difference between control group and video assisted induction group.

\begin{tabular}{cccccc}
\hline Group 1: 2 - 3 years old & Number & Age (year) & Body weight (kg) & Height (cm) & Heart rate at the ward (bpm) \\
\hline Control group & 12 & $3 \pm 1$ & $14 \pm 2$ & $92 \pm 6$ & $95 \pm 15$ \\
Video assisted induction group & 8 & $3 \pm 1$ & $13 \pm 2$ & $87 \pm 8$ & $96 \pm 12$ \\
Group 2: 4 - 6 years old & Number & Age (year) & Body weight (kg) & Height (cm) & Heart rate at the ward (bpm) \\
Control group & 12 & $5 \pm 1$ & $17 \pm 2$ & $107 \pm 4$ & $89 \pm 9$ \\
Video assisted induction group & 13 & $5 \pm 1$ & $17 \pm 3$ & $103 \pm 17$ & $90 \pm 17$ \\
Group 3: 7 - 10 years old & Number & Age (year) & Body weight (kg) & Height (cm) & Heart rate at the ward (bpm) \\
Control group & 14 & $8 \pm 1$ & $25 \pm 6$ & $127 \pm 12$ & $83 \pm 12$ \\
Video assisted induction group & 15 & $9 \pm 1$ & $27 \pm 5$ & $131 \pm 9$ & $87 \pm 18$ \\
\hline
\end{tabular}

Table 2. The kind of operation was listed.

Inguinal hernioplasty
Umbilicus hernioplasty
Orchiopexy
Pectus excavatum
Vesicoureteral backward flow
Ureteral stenosis
Muscle biopsy
Hypospadia formation


Group 1:2-3 years old

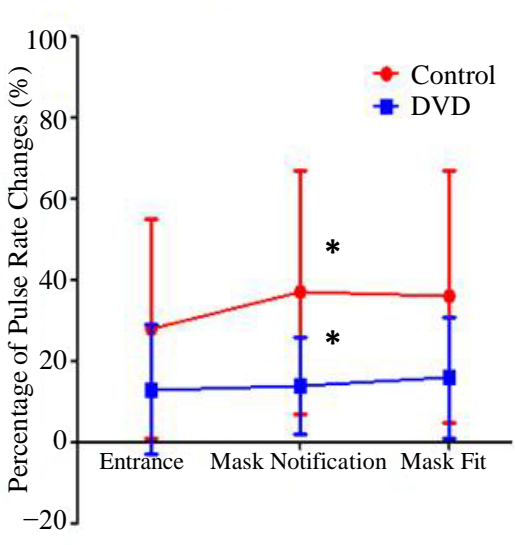

Group 2:4-6 years old

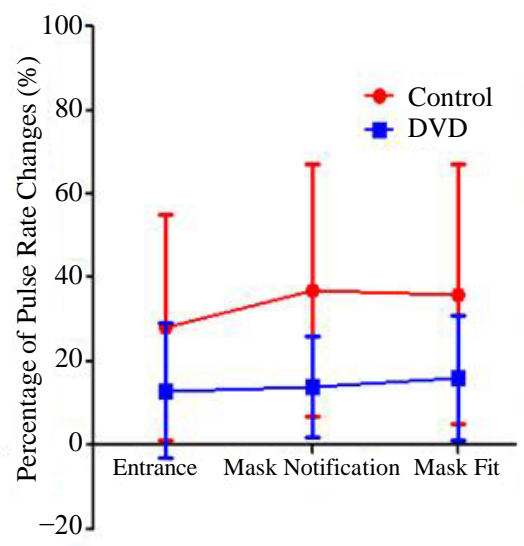

Group 3:7-10 years old

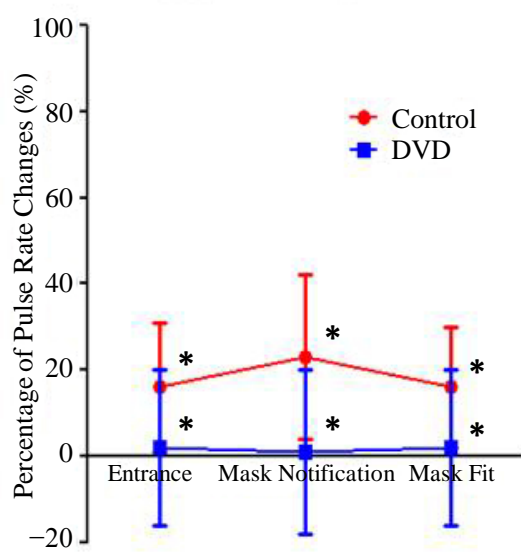

$* \mathrm{P}<0.05$ Control group vs. video assisted induction group in each group

Figure 1. These graphs showed that the changing of pulse rate in each group. The changing of pulse rate was calculated at each point (the entrance of operating room, when child saw the mask, and when the child was fit the mask) based on the pulse rate in the children's ward.

Group $1: 2$ - 3 years old

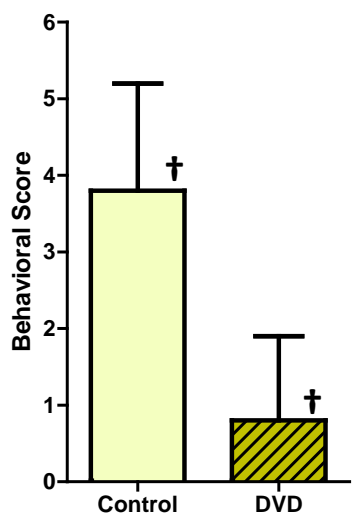

Group $2: 4$ - 6 years old

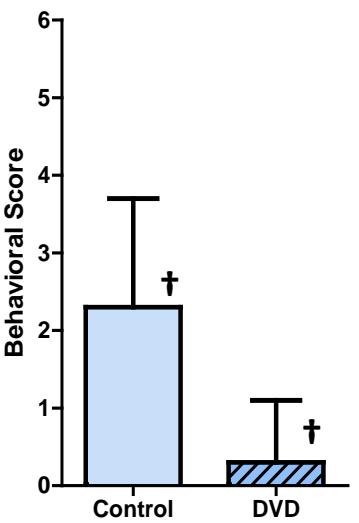

Group $3: 7$ - 10 years old

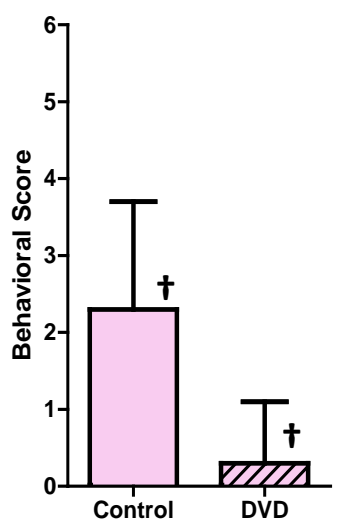

$+p<0.05$ Control group vs. video assisted induction group in each group

Figure 2. These bar graphs demonstrated that the behavioral score in each group. From at the entrance of operating room, anesthesiologist checked the behavioral score. The behavioral score was chosen the number of distress behaviors: crying, screaming, nonverbal resistance and verbal resistance as Chorney previously described. The score was from 0 point to 5 point.

Table 3. The table exhibited the usage of NSAIDs in control group and video assisted group.

\begin{tabular}{cccc}
\hline & NSAIDs & Total number & Percentage (\%) \\
\hline Control group & 5 & 38 & 13.2 \\
Video-assisted induction group & 6 & 36 & 16.7 \\
\hline
\end{tabular}

\section{Discussion}

We hypothesized that video assisted anesthesia induction would diminish children's stress. When people feel fear or something unpleasant, their heart rate usually increases. If we were able to reduce their stress, their heart rate would not increase. This is the first report describing not only the pulse rate but also the behavioral score in pediatric patients who received video assisted anesthesia induction. We have shown that the children's overall stress was reduced by the video assisted induction. 
Interestingly, the heart rate did not increase in control group 2 did not increase. This phenomenon was remarkable. Regarding to Maria Montessori, an Italian physician, children from 4 to 6 years old, could understand what nurses and medical stuff did. Aged from 4 to 6 years old was the perceptiveness period of Maria Montessori and was time full of curiosity such as the collection of the information. Children aged 4 to 6 years old could understand the explanation of what we did for the children smoothly and without fear. That is why the pulse rates in control group 2 did not increase. Children aged 4 to 6 years old could manage to control their stress based on the doctor's information. However, for children 7 to 10 years old, their understanding deepened. They thought or reflected deeply, so that they felt fear in the operating room and about the medical machine. On the other hand, if there was something that they were interested in, the video assisted anesthesia induction group could be stable in their mental comfort. At the age of 2 to 3 years old, this is the time when there is unease about non-daily life, so fear came to the fore even if they were with their parents. There was a significant difference regarding the pulse rate between the control group and the video assisted induction group in group 1when the children saw the face mask. In this group, the greatest stressor was the mask. The video assisted induction reduced this stress. Only a song or video took the child's mind off unexpected things in this group. Also, there was a huge standard deviation in this group.

Anesthesiologist excluded the patients who repeatedly underwent operations under general anesthesia because a great deal of general anesthesia might affect a child's behavioral and emotional disturbances. Barki concluded there was a risk of becoming anxious or depressed by repeated general anesthesia [3]. Yuki demonstrated that the Induction of anesthesia could be a very stressful period for a child and his family and could be associated with increased risk of psychological disturbances [4]. We thought that psychological condition of children, who received operation repeatedly, was difference. The previous anesthesia induction might be influence to the next one. It was known to the previous medical encounters were defined as children's factor.

MacLaren reported that more than $40 \%$ of children from two to ten year's old displayed distress during the anesthesia induction [2]. In addition, $42 \%$ of the time that the mask was placed, most children showed nonverbal resistance. Many non-pharmacological interventions were investigated. Manyande demonstrated the effects of non-pharmacological interventions in assisting the induction of anesthesia in children for reducing their anxiety, distress or increasing their co-operation [5]. They concluded that the presence of parents during induction of pediatric anesthesia did not diminish the child's anxiety. Also, most non-pharmacological interventions such as video-assisted induction, video games, the doctor as a clown, and low sensory induction could not be evaluated for whether they were effective or not, because the population was too small. However, outcome measurements of our research used an objective evaluation, which was the pulse rate accompanied by observation of the behavior. Still, the number in each group was small. Further investigation is needed, including parental anxiety.

When a video that the children' chose was played during induction, the children were significantly less anxious than the controls in a trial of 91 children [6]. In another trial, according to Berghmans of 120 children, co-operation at induction did not differ significantly when a video of a fairytale was played before induction [7]. Children exposed to low sensory stimulation were significantly less anxious than control children upon introduction of the anesthesia mask, and more likely to be co-operative during induction, in one trial of 70 children [8]. Music therapy did not show a significant effect on children's anxiety in another trial of 51 children [9].

Playing videos of the child's choice during induction, pre-operative hypnosis and hand-held video games all require further testing in future studies [10]. Non-drug interventions that might help the parents to relax also need further study, as there is some evidence that more relaxed parents may improve their child's anesthesia induction experience.

Evaluation of this research as pulse rate was the invariable at each point. Therefore, there was no difference in measurements based on the person that evaluated it. We used the number of distress score because it was easy to evaluate quickly, even if the anesthesiologist anesthetized pediatric patients. However, the limitation of this research was that we could not blind to measure the pulse rate and the behavioral score in each group. We should investigate the postoperative pain and the long-term psychological effect prospectively. Further investigation is needed.

In summary, these results showed that video assisted anesthesia induction helped decrease stress with regard to the increasing pulse rate and decreasing behavioral score in patients who are children.

\section{Conclusion}

These results showed that the video assisted anesthesia induction was effective in pediatric patients, especially 
those from 7 to 10 years old.

\section{Conflict of Interest Statement}

None of the authors or our institution has received any financial or material support from the manufacturer.

\section{Funding}

This study was supported in part by the university’s internal clinical research budget.

\section{References}

[1] Kain, Z.N., Mayes, L.C., Caldwell-Andrews, A.A., Karas, D.E. and McClain, B.C. (2006) Preoperative Anxiety, Postoperative Pain, and Behavioral Recovery in Young Children Undergoing Surgery. Pediatrics, 118, 651-658. http://dx.doi.org/10.1542/peds.2005-2920

[2] Chorney, J.M. and Kain, Z.N. (2009) Behavioral Analysis of Children's Response to Induction of Anesthesia. Anesthesia and Analgesia, 109, 1434-1440. http://dx.doi.org/10.1213/ane.0b013e3181b412cf

[3] Bakri, M.H., Ismail, E.A., Ali, M.S., Elsedfy, G.O., Sayed, T.A. and Ibrahim, A. (2015) Behavioral and Emotional Effects of Repeated General Anesthesia in Young Children. Saudi Journal of Anaesthesia, 9, 161-166. http://dx.doi.org/10.4103/1658-354X.152843

[4] Yuki, K. and Daaboul, D.G. (2011) Postoperative Maladaptive Behavioral Changes in Children. Middle East Journal of Anesthesiology, 21, 183-189.

[5] Manyande, A., Cyna, A.M., Yip, P., Chooi, C. and Middleton, P. (2015) Non-Pharmacological Interventions for Assisting the Induction of Anaesthesia in Children. The Cochrane Database of Systematic Reviews, 7, CD006447. http://dx.doi.org/10.1002/14651858.cd006447.pub3

[6] McEwen, A., Moorthy, C., Quantock, C., Rose, H. and Kavanagh, R. (2007) The Effect of Videotaped Preoperative Information on Parental Anxiety during Anesthesia Induction for Elective Pediatric Procedures. Pediatric Anesthesia, 17, 534-539. http://dx.doi.org/10.1111/j.1460-9592.2006.02173.x

[7] Berghmans, J., Weber, F., van Akoleyen, C., Utens, E., Adriaenssens, P., Klein, J., et al. (2012) Audiovisual Aid Viewing Immediately before Pediatric Induction Moderates the Accompanying Parents' Anxiety. Pediatric Anesthesia, 22, 386-392. http://dx.doi.org/10.1111/j.1460-9592.2011.03767.x

[8] Kain, Z.N., Wang, S.M., Mayes, L.C., Krivutza, D.M. and Teague, B.A. (2001) Sensory Stimuli and Anxiety in Children Undergoing Surgery: A Randomized, Controlled Trial. Anesthesia and Analgesia, 92, 897-903. http://dx.doi.org/10.1097/00000539-200104000-00018

[9] Kain, Z.N., Caldwell-Andrews, A.A., Krivutza, D.M., Weinberg, M.E., Gaal, D., Wang, S.M., et al. (2004) Interactive music Therapy as a Treatment for Preoperative Anxiety in Children: A Randomized Controlled Trial. Anesthesia and Analgesia, 98, 1260-1266. http://dx.doi.org/10.1213/01.ANE.0000111205.82346.C1

[10] Patel, A., Schieble, T., Davidson, M., Tran, M.C., Schoenberg, C., Delphin, E., et al. (2006) Distraction with a HandHeld Video Game Reduces Pediatric Preoperative Anxiety. Pediatric Anesthesia, 16, 1019-1027. http://dx.doi.org/10.1111/j.1460-9592.2006.01914.x 